\title{
Evaluation of YouTube Videos Regarding Clean Intermittent Catheterization Application
}

\author{
Yeliz Culha ${ }^{1}$, Mehmet Gokhan Culha², Rengin Acaroglu ${ }^{1}$ \\ ${ }^{1}$ Fundamentals of Nursing Department, Istanbul University-Cerrahpasa Florence Nightingale Faculty of Nursing, Istanbul, Turkey \\ ${ }^{2}$ Urology Department, University of Health Sciences, Okmeydani Training and Research Hospital, Istanbul, Turkey
}

Purpose: The aim of this study was to assess the clean intermittent catheterization (CIC) training and education videos on YouTube.

Methods: This study was conducted in English language in descriptive type to evaluate the content, reliability, and quality of Internet videos related to CIC training. The search was performed by using term "clean intermittent catheterization" and "intermittent self catheterization" on YouTube in August 2019. The content of the selected videos was analyzed by 2 independent experts in the field. The DISCERN questionnaire was used to analyze the reliability of the video and the global quality score (GQS) was used for the quality of the video.

Results: When the contents of the 64 videos included in the study were examined, it was found that 41 of them contained useful information and 23 of them contained misleading information. DISCERN scores, video coverage scores, and GQS of videos with useful information were found to be statistically higher than those with misleading information $(\mathrm{P}<0.001)$. When the sources of the videos were examined, it was seen that $78.05 \%$ of the videos containing useful information were published by medical advertisement/for-profit companies $(\mathrm{P}<0.001)$. The reliability scores, coverage scores, and GQS of the videos from medical advertisement/for-profit companies were significantly higher than the other sources $(\mathrm{P}<0.001)$.

Conclusions: In this study, it was seen that the majority of YouTube CIC training videos examined within the scope of the research were published by medical advertisement/for-profit companies and most of these videos contain useful information.

Keywords: Clean intermittent catheterization; YouTube; Training; Reliability; Usefulness

- Research Ethics: The study was approved by the Clinical Researches Ethics Committee of Istanbul University, Faculty of Dentistry with number 22/2019.

- Conflict of Interest: No potential conflict of interest relevant to this article was reported.

\section{INTRODUCTION}

Clean intermittent catheterization (CIC) is a method accepted as golden standard in individuals with bladder dysfunction and in the treatment of urinary retention [1]. CIC is considered as a safe and efficient method, which supports the maintenance of bladder functions by providing urinary excretion in individuals with neurogenic bladder, improves the body image and selfconfidence of the patient, and increases his/her life quality [2]. However, inappropriate catheterization may lead to the occurrence of CIC-related complications such as pain, urethral trauma, hematuria, and lower urinary tract infection [3]. CIC application training is crucial in bringing in the patient him/herself or his/her caregiver the knowledge and skills related to the 
application in the right way [4].

Various methods of education are used in CIC education, including verbal education, written material/brochures, simulation, and multimedia-based education [2]. It is seen that education methods applied by utilizing technology are becoming more and more widespread. Video-assisted education, which is among these methods, and which provides ease of learning to individuals with the visual and audio materials it contains, has been frequently preferred in clinical learning environment and patient education in recent years [5]. With the development of technology, the Internet has become an important source of health information. The ease of finding information on the Internet means responding to health/disease-related questions and concerns [6]. Especially programs that present knowledge and skills audio-visually are broadcast on different websites, mainly YouTube [7]. YouTube users composed $95 \%$ of all Internet users [8]. YouTube, which is one of the most visited websites with a total of more than a billion visitors per month, includes users' shares as well as reliable training videos [9]. YouTube has great potential to provide easy access to health-related information, but it is unknown how accurate and useful the information received [6].

Although studies report that YouTube videos are educational tools and sources of information for patients, the lack of studies examining whether patient training videos contain safe and accurate information/application skills is noteworthy, and these studies are confined to methotrexate injection, cervical cancer, rheumatoid arthritis, breast self-examination, and mammography training videos $[7,10]$. When national and international literature was examined, only one study was found regarding the evaluation of CIC videos published on websites as a reliable source of information [11]. Accordingly, in this study, it was aimed to examine the content, reliability, and quality of Internet videos related to CIC application training. It is thought that the results of the study will guide the creation of training videos for CIC application and will contribute to the increase of videos that will support sick individuals' acquisition of the correct skills regarding the application.

\section{Research Questions}

1. Is the content of YouTube videos about CIC training sufficient?

2. On what level is the reliability of YouTube videos about CIC training?

3. On what level is the quality of Internet videos about CIC ed- ucation?

4. What is the broadcast source of YouTube videos about CIC training?

\section{MATERIALS AND METHODS}

This study was carried out in a descriptive type to evaluate the content, reliability, and quality of the YouTube videos related to the CIC application.

All the videos broadcast on YouTube (http://www.youtube. com) until August 31, 2019, were watched as a result of the search with the words "clean intermittent catheterization" and "intermittent self catheterization" in English. A total of 140 videos added to the playlist automatically were reviewed according to inclusion criteria. Inclusion criteria: (1) videos broadcast in English, (2) videos demonstrating the ease of application, (3) the last updated version was determined for repeated videos. Seventy-six videos were excluded (50 videos that did not show the CIC application and were only informative, 8 videos, which were not in English, 18 repeated videos). The study was completed with the analysis of a total of 64 English videos (Fig. 1). The evaluation of videos in terms of reliability, quality, and scope of videos was performed by 2 independent persons. One of the observers evaluating the videos is a urology specialist who is interested in functional urology, especially neurogenic bladder patients and the other is a nurse who is particularly interested in CIC training.

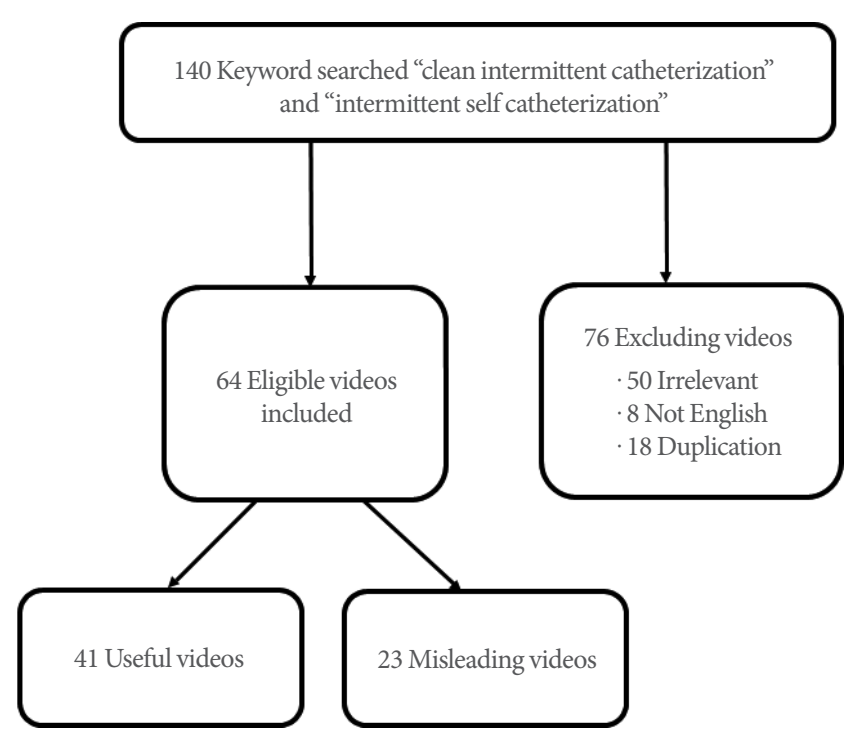

Fig. 1. Selection of eligible YouTube videos for the study. 


\section{Evaluation of Videos}

The contents of the videos were reviewed by 2 independent people and classified as useful and misleading: Useful videos; those with scientifically correct information regarding the CIC catheterization application, misleading videos; those which contain scientifically unproven information. The reliability of the agreement between the 2 independent observers was determined by calculating the Kappa value.

After the videos were watched, they were grouped as government/news agencies, universities/professional organizations/ nonprofit physician/physician groups, private websites with health information, medical advertisement/nonprofit companies, personal experiences. The time elapsed since the video was uploaded, the duration of the video and the number of views, likes, dislikes, comments of each video were recorded and the audience interaction was calculated. The reliability of the videos included in the study was evaluated with the DISCERN query tool, and their quality was evaluated with the global quality score (GQS). Its comprehensiveness was assessed by a list of skills created by researchers in line with international guidelines and involving CIC implementation steps.

\section{Comprehensiveness evaluation of videos}

The comprehensiveness of the videos was evaluated with a skill list of 8 items, prepared in line with the European Association of Urology Nurses [3] and Society of Urologic Nurses and Associates [12] CIC practical guides (Table 1) and the "yes" answer for each item in this skill list was evaluated as 1 point. The points that can be taken from the skill list are between $0-8$. It is accepted that the higher the total score is, the higher the overall video coverage will be.

\section{Evaluation of the reliability of videos (DISCERN)}

Regarding the evaluation of the reliability of the video, the short form of the DISCERN questioning pool, which Charnock and Shepperd [13] developed to evaluate written health information, adapted by Singh et al. [14] was used (Table 1). In this form, the yes answer given for each item was evaluated as 1 point. The scores that can be obtained from the inquiry form are between 0-5. In DISCERN scoring, videos greater than 3 are good quality videos and contain useful information for the patient. Videos with a score of 3 are considered medium quality and require additional sources of information. Videos with a score less than 3 should be considered bad quality and should not be used by patients.
Table 1. Reliability, quality and comprehensiveness assessment tools of internet videos for clean intermittent catheterization training

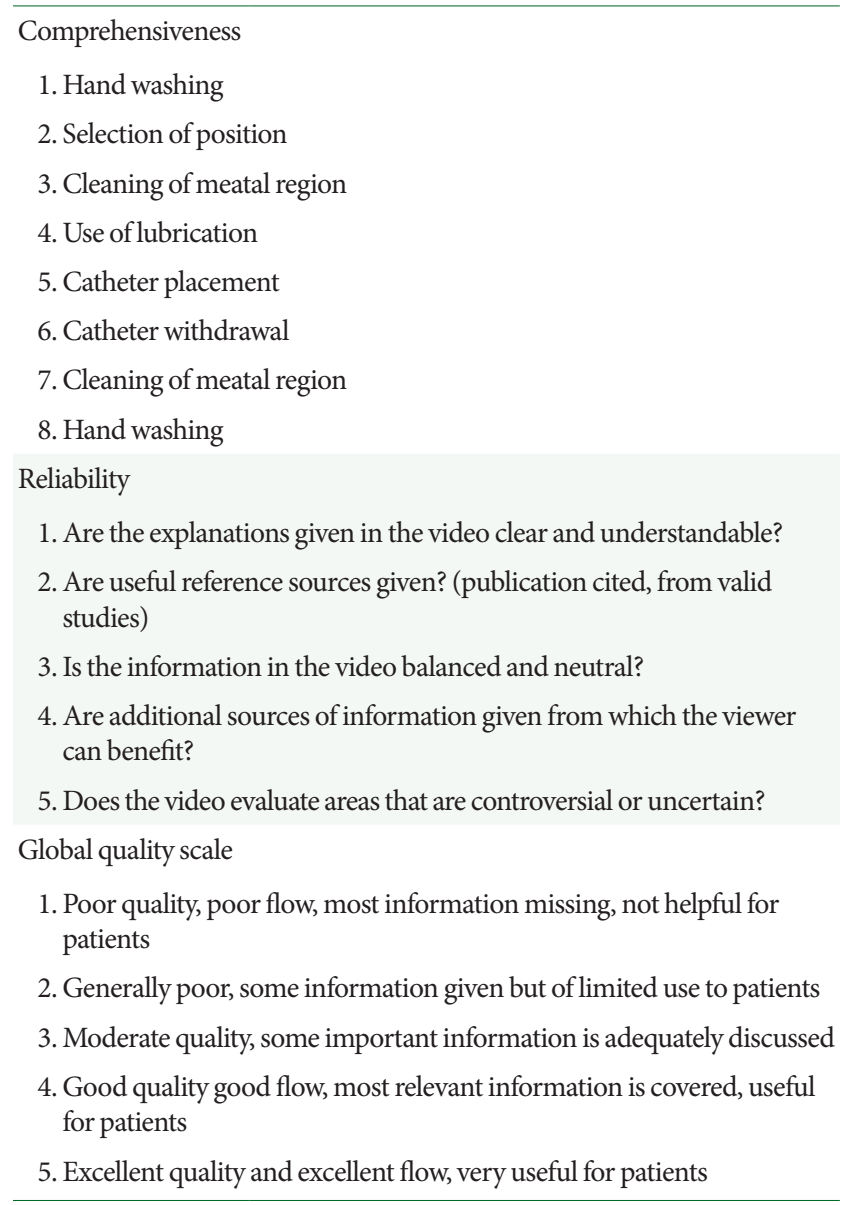

\section{Evaluation of the quality of videos}

The overall quality of the videos was evaluated with a 5-point GQS, which Bernard et al. [15] developed to evaluate the flow of information and videos on the website, their quality, these sources' ease of use for the patient and how useful they will be (Table 1). There are 5 items, which evaluate the quality of the video in the form (1: poor quality, 2: low quality - limited use, 3 : somewhat useful, 4: useful, 5: useful-excellent quality). In the evaluation, the item that best expresses the quality of the video is selected.

\section{The Ethical Aspect of the Research}

The names of the people/organizations who prepared the video were kept confidential. The study was approved by the Clinical Researches Ethics Committee of Istanbul University, Faculty of Dentistry with number 22/2019. 


\section{Statistical Analysis}

IBM SPSS Statistics ver. 20.0 (IBM Co., Armonk, NY, USA) was used to evaluate the data. The Kappa value was calculated and determined for the compatibility between the 2 independent observers. The distribution of the variables was measured by the Kolmogorov-Smirnov test. Comparison between groups was evaluated by independent sample t-test, Mann-Whitney Utest, chi-square test, and analysis of variance. Statistical significance level was accepted as $\mathrm{P}<0.05$.

\section{RESULTS}

When the content of the 64 videos included in the study was examined by 2 independent individuals, it was seen that 41 of the videos (64.06\%) were useful and 23 (35.94\%) were misleading. The Kappa value among the observers was 0.96 . The total duration of useful videos on YouTube was determined as $37.49 \pm 24.67$ months, the total length as $325.59 \pm 271.87 \mathrm{sec}-$ onds, the number of views as $31,867.93 \pm 90,926.32$, the number of likes as $44.61 \pm 102.51$, the number of dislikes as $9.10 \pm 24.54$, and the number of comments as $3.05 \pm 8.71$. Forty-one point forty-six percent (41.46\%) of the useful videos showed application skill in female patients, $41.46 \%$ in male patients, $2.43 \%$ in both sexes, and $14.63 \%$ in children. When misleading videos were analyzed, it was seen that the total duration of finding videos on YouTube was $33.78 \pm 27.88$ months, the total length $306.39 \pm 246.25$ seconds, the number of views $11,371.83 \pm$ $18,512.02$, the number of likes $20.83 \pm 26.94$, the number of dislikes $3.04 \pm 4.43$, and the number of comments $4.26 \pm 7.54$ (Table 2). Thirty-four point seventy-eight percent (34.78\%) of misleading videos showed CIC skill regarding female patients, $56.52 \%$ regarding male patients, and $8.7 \%$ regarding both sexes
(Table 2).

When videos are analyzed, the mean score of useful videos was found to be $6.43 \pm 1.36$, DISCERN mean score $4.12 \pm 0.97$, and the mean GQS $4.09 \pm 1.01$. On the other hand, the comprehensiveness mean score of the misleading videos was $2.87 \pm 0.92$, DISCERN mean score $1.85 \pm 0.65$, and the mean GQS $1.78 \pm$ 0.62 (Table 3 ). There was no statistically significant difference between useful videos and misleading videos in terms of video length, number of views, likes, dislikes, and the number of comments. On the other hand, the mean comprehensiveness scores, mean DISCERN scores, and mean GQS of useful videos were found to be higher than misleading videos, with a statistically significant difference $(\mathrm{P}<0.001)$ (Table 3$)$. The features of the 10

Table 2. Characteristics of YouTube videos

\begin{tabular}{lccc}
\hline Characteristic & $\begin{array}{c}\text { Useful video } \\
(\mathrm{n}=41)\end{array}$ & $\begin{array}{c}\text { Misleading video } \\
(\mathrm{n}=23)\end{array}$ & P-value \\
\hline $\begin{array}{l}\text { Duration on } \\
\text { YouTube }(\mathrm{mo})\end{array}$ & $37.49 \pm 24.67$ & $33.78 \pm 27.88$ & \\
$\begin{array}{c}\text { Video length } \\
(\mathrm{sec})\end{array}$ & $325.59 \pm 271.87$ & $306.39 \pm 246.25$ & 0.780 \\
Total views & $31,867.93 \pm 90,926.32$ & $11,371.83 \pm 18,512.02$ & 0.584 \\
Views per day & & & \\
Likes & $44.61 \pm 102.51$ & $20.83 \pm 26.94$ & 0.281 \\
Dislikes & $9.10 \pm 24.54$ & $3.04 \pm 4.43$ & 0.247 \\
Comments & $3.05 \pm 8.71$ & $4.26 \pm 7.54$ & 0.578 \\
Sex & & & 0.140 \\
Female & $17(41.46)$ & $8(34.78)$ & \\
Male & $17(41.46)$ & $13(56.52)$ & \\
Both & $1(2.43)$ & $2(8.7)$ & \\
Children & $6(14.63)$ & $0(0)$ & \\
\hline
\end{tabular}

Values are presented as mean \pm standard deviation or number (\%).

Table 3. Analysis of videos useful and misleading

\begin{tabular}{|c|c|c|c|}
\hline Characteristic & Useful video $(n=41)$ & Misleading video $(\mathrm{n}=23)$ & P-value \\
\hline Reliability score & $4.12 \pm 0.97$ & $1.85 \pm 0.65$ & $<0.001^{*}$ \\
\hline Global quality score & $4.09 \pm 1.01$ & $1.78 \pm 0.62$ & $<0.001^{*}$ \\
\hline Comprehensiveness score & $6.43 \pm 1.36$ & $2.87 \pm 0.92$ & $<0.001^{\star}$ \\
\hline Source of upload & & & $<0.001^{*}$ \\
\hline Universities/professional organizations/nonprofit physician/physician groups & $5(12.19)$ & $4(17.39)$ & \\
\hline Stand-alone health information websites & $4(9.76)$ & $0(0)$ & \\
\hline Medical advertisement/for profit companies & $32(78.05)$ & $9(39.13)$ & \\
\hline Individual & $0(0)$ & $10(43.48)$ & \\
\hline
\end{tabular}

Values are presented as mean \pm standard deviation or number (\%).

${ }^{\star}$ Independent sample t-test. 
Table 4. Analysis of videos by source of uploads

\begin{tabular}{|c|c|c|c|c|c|}
\hline Variable & $\begin{array}{l}\text { Universities/professional } \\
\text { organizations/nonprofit } \\
\text { physician/physician groups }\end{array}$ & $\begin{array}{l}\text { Stand-alone health } \\
\text { information websites }\end{array}$ & $\begin{array}{l}\text { Medical advertisement/ } \\
\text { for profit companies }\end{array}$ & Individual & P-value \\
\hline Video number & $9(14.06)$ & $4(6.25)$ & $41(64.06)$ & $10(15.63)$ & \\
\hline Reliability score & $2.39 \pm 1.24$ & $2.63 \pm 0.63$ & $4.00 \pm 1.09$ & $1.55 \pm 0.50$ & $<0.001$ \\
\hline Comprehensiveness score & $4.11 \pm 1.75$ & $4.38 \pm 0.75$ & $6.11 \pm 1.77$ & $2.45 \pm 0.69$ & $<0.001$ \\
\hline Global quality score & $2.39 \pm 1.29$ & $2.63 \pm 063$ & $3.98 \pm 1.06$ & $1.35 \pm 0.41$ & $<0.001$ \\
\hline Total view & $95,268.22 \pm 179,976.48$ & $2,756.50 \pm 2,133.05$ & $15,176.98 \pm 27,683.99$ & $7,744.10 \pm 11,996.22$ & 0.017 \\
\hline Video length (sec) & $559.33 \pm 510.52$ & $247.00 \pm 193.31$ & $246.12 \pm 116.44$ & $428.30 \pm 274.61$ & 0.003 \\
\hline Duration on YouTube (mo) & $65.78 \pm 33.90$ & $69.00 \pm 27.54$ & $30.54 \pm 16.09$ & $19.40 \pm 19.73$ & $<0.001$ \\
\hline Likes & $88.00 \pm 168.69$ & $4.00 \pm 3.65$ & $28.22 \pm 66.01$ & $34.30 \pm 36.14$ & 0.225 \\
\hline Dislikes & $26.00 \pm 47.88$ & $1.00 \pm 0.21$ & $4.24 \pm 8.21$ & $3.10 \pm 4.33$ & 0.018 \\
\hline Comments & $26.00 \pm 47.88$ & $1.00 \pm 0.21$ & $4.24 \pm 8.21$ & $3.10 \pm 4.33$ & 0.073 \\
\hline Misleading information & $4(44.44)$ & $0(0)$ & $9(21.95)$ & $10(100)$ & $<0.001$ \\
\hline Useful information & $5(55.56)$ & $4(100)$ & $32(78.05)$ & $0(0)$ & $<0.001$ \\
\hline
\end{tabular}

Values are presented as number (\%) or mean \pm standard deviation.

most useful videos according to their quality and suitability are indicated in the Supplementary Table 1.

When the videos were analyzed according to publication sources, $78.05 \%$ of the videos ( 32 of 41 ) were found to be published by medical advertisement/for-profit companies (Table 3). It was seen that $43.48 \%$ of misleading videos consisted of videos with individual sources and that no useful videos were included in the videos with individual sources (Table 4). The mean scores of videos originating from medical advertisement/for-profit companies, their mean DISCERN scores, and mean GQS were found to be statistically significantly higher than other broadcast-originated videos $(\mathrm{P}<0.001)$ (Table 4$)$. The average number of views and average video duration of the videos originating from universities/professional organizations/nonprofit physician/physician groups were found to be significantly higher than the videos from other sources $(\mathrm{P}=0.017$ for the number of views, $\mathrm{P}=0.003$ for video duration).

\section{DISCUSSION}

Main objective is to provide low intravesical pressure and maintain upper urinary system function in the treatment of neurogenic bladder. In line with these goals, quality of life is improved [16]. In patients with neurogenic bladder, CIC is performed with an interval of 4-6 hours in order to protect the bladder and upper urinary system. Despite the ease of application, intermittent catheterization training is considered a problem by pa- tients, families, and healthcare professionals $[17,18]$. Poor or inadequate "catheterization" technique can cause complications such as urinary tract infection and trauma, pain and discomfort, urethral stenosis, and false urethral canal [19]. Therefore, learning in a virtual environment allows flexibility and expansion of information and information access, cooperates with the formation of groups of common interests, overcomes physical evidence, or difficulty in accessing scientific evidence [20, 21].

There is a range of information on health-related issues (professional opinions, literature information, innovations in the field of health, individual health experiences, comments of nonspecialists, etc.) on the Internet. This information is increasingly used by patients and healthcare professionals as an educational resource [22]. Founded in September 2005, YouTube is an open access, video-sharing website, which also includes information and educational videos aimed at individuals. Since being auditory and visual are important factors in accessing information and due to the ease of accessing information, YouTube's popularity among patients has been gradually increasing $[7,23]$. However, that everyone can easily upload videos renders the inspection of the reliability and the accuracy, which leads to the presentation of missing or incorrect information [7]. The existence of unreliable information, especially in training videos aimed at bringing in skills can mislead patients and lead to undesired consequences due to misapplication. Inappropriate application of CIC may lead to complications such as urethral 
trauma, hematuria, and urinary tract infections, which may adversely affect the individual's quality of life [2,3].

Within the scope of this study, only 6 showed the skills regarding CIC application for children (Table 2). During the lifespan of the individual, childhood is evaluated together with physiological and requires a psychological development and a CIC education that will meet their cognitive development. This finding suggests that the number of CIC training videos for children is insufficient and that effective, accurate, and comprehensive videos that can be utilized by children should be made available by health professionals.

As seen in Table 2, the majority of the total videos consisted of useful videos (64.06\%) (Table 2). When the analysis results of the videos were examined, it was determined that useful videos contained comprehensive, reliable, and useful information, whereas misleading videos contained insufficient, unreliable, and low-quality information (Table 3). However, the total number of views, likes, and dislikes of useful videos were found to be higher than that of misleading videos (Table 2). This finding was found to be similar to the findings of studies examining rheumatoid arthritis, subcutaneous antitumor agent administration, and self-breast examination training videos on YouTube $[7,8,14]$. At the same time, this finding showed that the patients who performed CIC preferred the more comprehensive and reliable training videos.

Whereas only 5 of the useful videos (12.19\%) were broadcast based on universities/professional organizations/nonprofit physician/physician groups, 32 of them (78.05\%) were broadcast based on medical advertisement/for-profit companies. While this finding, which revealed that very few of the useful videos were broadcast by universities/professional organizations/nonprofit physician/physician groups, showed similarity with the results of the study in which Faleiros et al. [11] examined the CIC skill training videos in Portuguese language, it differed from the results of the study where self-breast examination and subcutaneous antitumor agent application training videos were examined $[7,8]$. Regarding comprehensiveness point averages, DISCERN point averages and GQS point averages, videos originating from medical advertisement/for-profit companies were found to contain safe and accurate information for CIC skill training and were of high quality (Table 4). However, it was observed that the vast majority of these videos originating from the medical advertisement/for-profit companies were published by the catheter companies, and in the vast majority of these videos, their application skills were presented together with the features that emphasized firms' use of their own catheters. This situation suggested that it may cause anxiety in individuals using different types of catheters.

While 4 of the misleading videos (17.39\%), which constitute $35.9 \%$ of the total videos were originated from universities/professional organizations/nonprofit physician/physician groups, it was found that the broadcast source of 9 of them (39.13\%) was medical advertisement/for-profit companies and 10 of them (43.48\%) individual. In terms of mean DISCERN score, mean GQS, and mean comprehension score, these videos were found to comprise unreliable, insufficient, or inaccurate information for CIC training and to be of poor quality. This finding was similar to the results of the study examining videos about subcutaneous antitumor agent administration and health information and self-methotrexate injection training for patients with type 2 diabetes $[8,10,24]$. This finding also reveals the need for the preparation of training videos regarding CIC led by state institutions/organizations and the presentation of information that will meet the needs of patients on the web.

It is a limitation of the study that the demographic characteristics of the video viewers are not known.

In this study, it was seen that the vast majority of YouTube videos aimed at CIC training consist of useful videos and published by medical advertisement/for-profit companies. When assessed against internationally recognized standards, the majority of high-quality videos are published by commercial organization, promoting the use of their product. There is a dearth of information for children. Videos produced by individuals tend to be of lower quality and are more likely to contain misleading information. In line with these results, it may be suggested that studies to increase the number of accurate, reliable, and useful videos that will bring in CIC-specific knowledge and skills are performed by healthcare professionals led by governmental institutions, universities, and professional organizations.

\section{SUPPLEMENTARY MATERIAL}

Supplementary Table 1 can be found via https://doi.org/10.5213/ inj.2040098.049.

\section{AUTHOR CONTRIBUTION STATEMENT}

- Conceptualization: $M G C$

- Data curation: $Y C$

- Formal analysis: $Y C$ 
- Methodology: MGC

- Project administration: $Y C$

- Writing-original draft: $M G C$

- Writing-review \& editing: $R A$

\section{REFERENCES}

1. Newman DK, Willson MM. Review of intermittent catheterization and current best practices. Urol Nurs 2011;31:12-28, 48.

2. Silva DRA, Mazzo A, Jorge BM, Souza Júnior VD, Fumincelli L, Almeida RGS. Intermittent urinary catheterization: the impact of training on a low-fidelity simulator on the self-confidence of patients and caregivers. Rehabil Nurs 2017;42:97-103.

3. Lauridsen S, Cobussen-Boekhorst H, Eikenboom J, Geng V, Holroyd S, Lester M, et al., editors. European Association of Urology Nurses evidence-based guidelines for best practice in urological health care catheterisation urethral intermittent in adults dilatation, urethral intermittent in adults evidence-based guidelines for best practice in urological health care catheterisation urethral intermittent in adults dilatation, urethral intermittent in adults. ARNHEM (The Netherlands): European Association of Urology Nurses; 2013.

4. Biaziolo CFB, Mazzo A, Martins JCA, Jorge BM, Batista RCN, Tucci SJ Júnior. Validation of self-confidence scale for clean urinary intermittent self-catheterization for patients and health-caregivers. Int Braz J Urol 2017;43:505-11.

5. Mackay BJ, Anderson J, Harding T. Mobile technology in clinical teaching. Nurse Educ Pract 2017;22:1-6.

6. Drozd B, Couvillon E, Suarez A. Medical YouTube videos and methods of evaluation: literature review. JMIR Med Educ 2018; 4:e3.

7. Esen E, Aslan M, Sonbahar BÇ, Kerimoğlu RS. YouTube English videos as a source of information on breast self-examination. Breast Cancer Res Treat 2019;173:629-35.

8. Tolu S, Yurdakul OV, Basaran B, Rezvani A. English-language videos on YouTube as a source of information on self-administer subcutaneous anti-tumour necrosis factor agent injections. Rheumatol Int 2018;38:1285-92.

9. Basch CH, Hillyer GC, MacDonald ZL, Reeves R, Basch CE. Characteristics of YouTubeTM videos related to mammography. J Cancer Educ 2015;30:699-703.

10. Rittberg R, Dissanayake T, Katz SJ. A qualitative analysis of methotrexate self-injection education videos on YouTube. Clin Rheumatol 2016;35:1329-33.

11. Faleiros F, Silveira L, Cucick C, Cordeiro A, Kappler C. Analysis of
Brazilian videos for learning of bladder intermittent self-catheterization. Urol Nurs 2019;39:193-7.

12. Society of Urologic Nurses and Associates (SUNA). Clinical practice guidelines: adult clean intermittent catheterization. [Internet]. Pitman (NJ): Society of Urologic Nurses and Associates; 2006 [cited 2019 Nov 22]. Available from: http://www.suna.org/resources/adult CIC Guide. pdf.

13. Charnock D, Shepperd S. Learning to DISCERN online: applying an appraisal tool to health websites in a workshop setting. Health Educ Res 2004;19:440-6.

14. Singh AG, Singh S, Singh PP. YouTube for information on rheumatoid arthritis--a wakeup call? J Rheumatol 2012;39:899-903.

15. Bernard A, Langille M, Hughes S, Rose C, Leddin D, Veldhuyzen van Zanten S. A systematic review of patient inflammatory bowel disease information resources on the World Wide Web. Am J Gastroenterol 2007;102:2070-7.

16. Drake MJ. Management and rehabilitation of neurologic patients with lower urinary tract dysfunction. Handb Clin Neurol 2015; 130:451-68.

17. Le Breton F, Guinet A, Verollet D, Jousse M, Amarenco G. Therapeutic education and intermittent self-catheterization: recommendations for an educational program and a literature review. Ann Phys Rehabil Med 2012;55:201-12.

18. Moore KN, Fader M, Getliffe K. Long-term bladder management by intermittent catheterisation in adults and children. Cochrane Database Syst Rev 2007;(4):CD006008.

19. Bruschini H, Almeida FG, Srougi M. Upper and lower urinary tract evaluation of 104 patients with myelomeningocele without adequate urological management. World J Urol 2006;24:224-8.

20. McMullan M. Patients using the Internet to obtain health information: how this affects the patient-health professional relationship. Patient Educ Couns 2006;63:24-8.

21. Knight E, Intzandt B, MacDougall A, Saunders TJ. Information seeking in social media: a review of youtube for sedentary behavior content. Interact J Med Res 2015;4:e3.

22. Adhikari J, Sharma P, Arjyal L, Uprety D. YouTube as a source of information on cervical cancer. N Am J Med Sci 2016;8:183-6.

23. Erdem MN, Karaca S. Evaluating the accuracy and quality of the information in kyphosis videos shared on YouTube. Spine (Phila Pa 1976) 2018;43:E1334-9.

24. Leong AY, Sanghera R, Jhajj J, Desai N, Jammu BS, Makowsky MJ. Is YouTube useful as a source of health information for adults with type 2 diabetes? A South Asian Perspective. Can J Diabetes 2018; 42:395-403.e4. 